\title{
Pathogenicity of Pepper mild mottle virus Is Controlled by the RNA Silencing Suppression Activity of Its Replication Protein but Not the Viral Accumulation
}

\author{
Shinya Tsuda, Kenji Kubota, Ayami Kanda, Takehiro Ohki, and Tetsuo Meshi
}

First, third, and forth authors: National Agricultural Research Center, Tsukuba, Ibaraki 305-8666, Japan; second author: National Agricultural Research Center for Kyushu Okinawa Region, Koshi, Kumamoto 861-1192, Japan; and fifth author: National Institute of Agrobiological Sciences, Tsukuba, Ibaraki 305-8602, and CREST, JST, Kawaguchi 322-0012, Japan. Accepted for publication 6 October 2006.

\begin{abstract}
Tsuda, S., Kubota, K., Kanda, A., Ohki, T., and Meshi, T. 2007. Pathogenicity of Pepper mild mottle virus is controlled by the RNA silencing suppression activity of its replication protein, but not the viral accumulation. Phytopathology 97:412-420.

Pepper mild mottle virus (PMMoV) infects pepper plants, causing mosaic symptoms on the upper developing leaves. We investigated the relationship between a virus pathogenicity determinant domain and the appearance of mosaic symptoms. Genetically modified PMMoV mutants

was relatively high. Conversely, the pseudoknot mutants showed the lower accumulation, but they still caused mosaic symptoms as severe as the wild-type virus. Therefore, the level of virus accumulation in a plant does not necessarily correlate with the development of mosaic symptoms. The activity to suppress posttranscriptional gene silencing (PTGS) was impaired in the asymptomatic mutant. Consequently, pathogenicity causing mosaic symptoms should be controlled by combat between host PTGS and its suppression by the $130 \mathrm{~K}$ replication protein rather than virus accumulation.
\end{abstract} were constructed, which had a base substitution in the $130 \mathrm{~K}$ replication protein gene causing an amino acid change or a truncation of the $3^{\prime}$ terminal pseudoknot structure. Only one substitution mutant (at amino acid residue 349) failed to cause symptoms, although its accumulation
Additional keywords: attenuated virus, cross protection, GFP, methyl bromide.

Tomato mosaic virus (ToMV) $\mathrm{L}_{11} \mathrm{~A}$ is an attenuated strain that has been used extensively to protect tomato plants from infection by virulent strains during cultivation in Japan (26). $\mathrm{L}_{11} \mathrm{~A}$ multiplies similarly to the parent wild-type strain ToMV L in protoplasts and in plants at earlier stages of infection, whereas the final accumulation of $\mathrm{L}_{11} \mathrm{~A}$ is $\approx 20 \%$ of that seen in wild-type ToMV infection $(16,25,39)$. Nucleotide sequence comparison between the $\mathrm{L}$ and $\mathrm{L}_{11} \mathrm{~A}$ strains found three base substitutions causing amino acid changes in the $130 \mathrm{k}$ replication protein (24). When these base changes were introduced into the L strain, the resulting virus exhibited the same attenuated phenotype as the $\mathrm{L}_{11}$ A strain (18).

Posttranscriptional gene silencing (PTGS), a homologydependent RNA degradation system that occurs in the cytoplasm, producing 21- to 25-nucleotide (nt) small interfering RNAs (13), functions to protect the plant against virus infection, whereas most plant viruses encode a suppressor to combat this defense (37). In the case of tobamoviruses, the $130 \mathrm{~K}$ replication protein has the suppressor function $(5,18)$. Recently, one of the three mutations found in the $\mathrm{L}_{11} \mathrm{~A}$ strain has been shown to be associated with a lowered suppressor activity and asymptomatic infection (18). Accordingly, a correlation exists between development of the systemic mosaic symptoms and the impaired activity of PTGS suppressor, but it has not been clarified whether the attenuated phenotype of $\mathrm{L}_{11} \mathrm{~A}$ is a direct result of the defect in the suppressor function or an indirect result of its lower accumulation.

The structure of the Tobamovirus 3' untranslated region (UTR) is divided into two subregions: one forming a tRNA-like structure (34) and the other comprising three to five consecutive pseudoknots just upstream from the tRNA-like structure $(9,10,28,36)$. This region appears to functionally substitute for a poly (A)

doi:10.1094/PHYTO-97-4-0412

(C) 2007 The American Phytopathological Society 
region in promoting interactions between the $5^{\prime}$ and $3^{\prime}$ termini, enhancing translation initiation in a cap-dependent manner (19). When nucleotide deletions were made in the central doublehelical segment of the three pseudoknots of the ToMV genome, viral multiplication was reduced significantly, accompanied by the loss of mosaic symptoms on tobacco plants (31).

In this study, we examined, based on the findings obtained from the earlier studies of ToMV mutants, whether the replication protein gene mutations and 3' UTR mutations are similarly effective to generate attenuated strains of PMMoV. Results showed that a replication protein mutant with a mutation resulting in the impairment of the suppressor function infects pepper plants systemically without causing visible symptoms. On the other hand, 3' UTR mutants infected pepper plants developing mosaic symptoms, unlike the case of ToMV. Accumulation levels of the mutant viruses in the upper leaves were not correlated with the severity of the visible symptoms.

\section{MATERIALS AND METHODS}

Plasmid constructions. pTPC4350 contains a full-length PMMoV cDNA, which was inserted downstream of a T7 promoter between the EcoRI and BamHI sites of pUC19. The CP gene sequence in pTPC4350 was derived from PMMoV-Ij, a strain overcoming the $L^{3}$ Tobamovirus resistance gene of pepper plants, and the other portions of the PMMoV sequence were from PMMoV-J $(17,35)$.

pTPC4350 was used as the starting material to produce two viruses with decreased pathogenicity by site-directed mutagenesis, using the sequence information provided by the ToMV $\mathrm{L}_{11} \mathrm{~A}$ genome (24). To introduce the first amino acid substitution, residue 348 , in the PMMoV $130 \mathrm{~K}$ replication protein, the sequence extending from the EcoRI site (upstream of the T7 promoter) to position 1670 in pTPC4350 was amplified as two DNA fragments by polymerase chain reaction (PCR). The mutations introduced are underlined within the following oligonucleotide primers. The front PCR fragment was amplified using the forward primer (nucleotides 344 to 363 of pUC19) and the reverse primer 5'-dAGGATTCTTTCGCTATT GTACATCGCCAAAGTCTTTTTGT (complementary to nucleotides 1091 to 1130 of pTPC4350). The rear PCR fragment was amplified with the forward primer 5'-dACAAAAAGACTTTGGCGATGTACAATAGCGAAAGAATCCT (corresponding to nucleotides 1091 to 1130 of pTPC4350) and the reverse primer 5'-dTTAACCAATCTATCGTGGAA (complementary to nucleotides 1651 to 1670 of pTPC4350). Finally, the PCR fragment for the mutation at residue 348 was amplified using the front and rear PCR fragments as templates with the forward primer (nucleotides 344 to 363 of pUC19) and the reverse primer (complementary sequence to nucleotides 1651 to 1670 of pTPC4350 above). The mutated fragment, confirmed its sequence, was excised using EcoRI and Eco47III (nucleotide 1308) and the corresponding fragment in pTPC4350 was replaced with the mutated fragment, the new plasmid being named pTPJ. The second amino acid substitution in the $130 \mathrm{~K}$ replication protein was created using the same strategy. The front PCR fragment was amplified using the forward primer (nucleotides 1131 to 1150 of pTPC4350) and the reverse primer 5'-dCACACCCCAAGCATGGTCTTTTGATAGAGGTTTCACCAAC (complementary to nucleotides 2331 to 2370 of pTPC4350), where mutations are underlined. The rear PCR fragment was amplified using forward primer 5'-dGTTGGTGAAACCTCTATCAAAA $\underline{\text { GACCATGCTT- }}$ GGGGTGTG (corresponding to nucleotides 2331 to 2370 of pTPC4350) and the reverse primer 5'-dTCTCCATAAACAAAAGCCTC (complementary to nucleotides 2851 to 2870 of pTPC4350). The mutated fragment was excised using Eco47III (nucleotide 1308) and SacI (nucleotide 2463), with which the corresponding fragment in pTPC4350 was replaced to create pTPA.
To introduce deletions into the pseudoknots of PMMoV RNA (Fig. 1, illustration for TPC4350), the nucleotide sequence extending from position 6044 to the $3^{\prime}$ terminus of pTPC4350 was amplified by PCR into two separate fragments. The first mutant had segments VI and V removed, from nucleotides 6177 to 6197 , and was named pTPD3-6197. The front PCR fragment, (as in all four mutants) was prepared with the forward primer (nucleotides 6044 to 6064 of pTPC4350, with an additional 5' terminal EcoRI site) and the reverse primer (complementary to nucleotides 6144 to 6176 of pTPC4350). The rear PCR fragment was amplified with forward primer (nucleotides 6144 to 6176 concatenated to nucleotides 6198 to 6230 ) and the reverse primer (nucleotides 6338 to the $3^{\prime}$ terminus of PMMoV). Using both the front and rear PCR fragments as templates, the corresponding sequences were amplified to form a single fragment using the forward primer of the front PCR fragment and the reverse primer for the rear PCR fragment. In the same strategy, the plasmid pTPD36207 was created by deleting nucleotides 6177 to 6207 , which correspond to segments VI, V, and IV. The third mutant plasmid, pTPD3-6219, was made carrying only double-helical segments II and I. The fourth plasmid, pTPD3-6249, had all the doublehelical segments deleted. All of the final products were digested using SfiI (site at nucleotide 6044) and BamHI, and gel purified, with which the corresponding fragment of pTPC4350 was replaced.
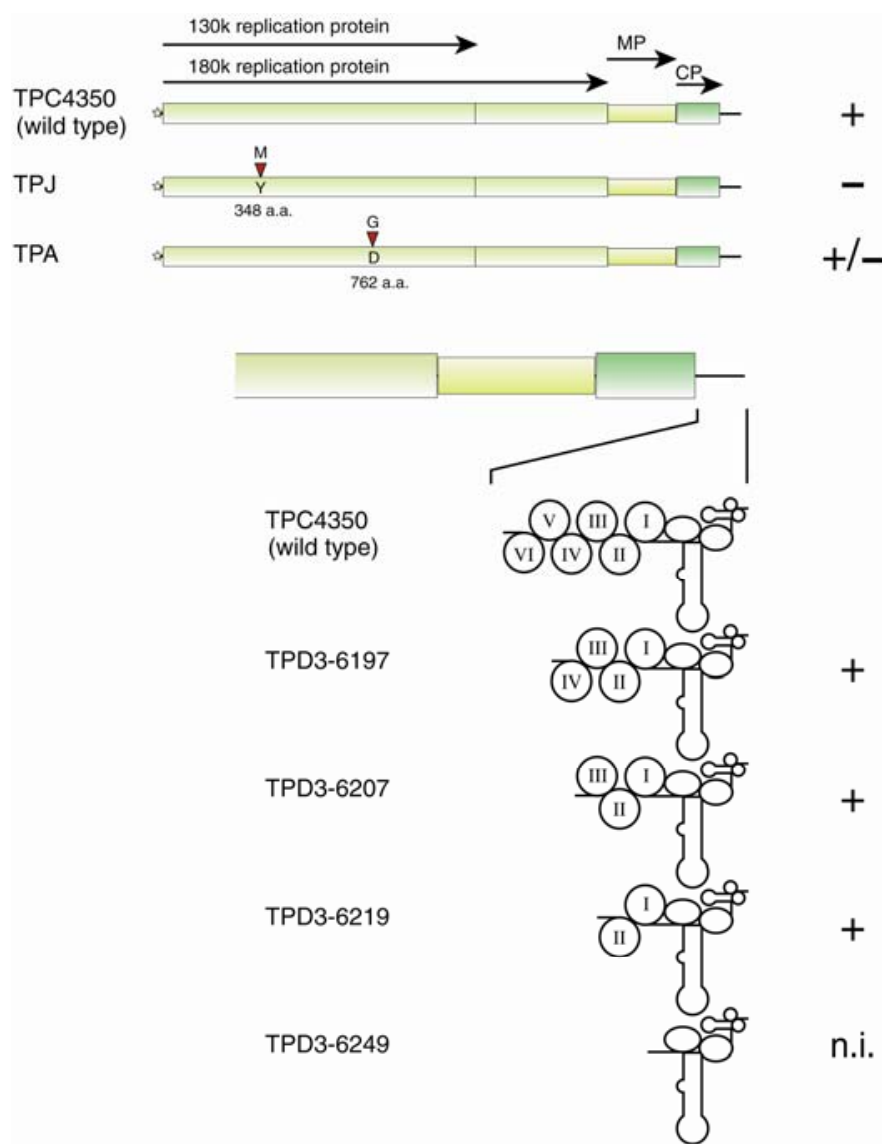

Fig. 1. Mapping the mutation or deletion responsible for the mosaic symptomdefective phenotype of Pepper mild mottle virus (PMMoV). The genomic structures of PMMoV and its derivatives are schematically shown. Amino acids differing from wild-type PMMoV (TPC4350) are shown with red closed triangles. "M" and "G" on each schematic construct indicates methionine and glycine as the original amino acid residues of wild-type PMMoV, respectively. "Y" and "D" in the constructs indicates tyrosine and aspartic acid, respectively, as residues substituted for an original one according to the sequence of ToMV $\mathrm{L}_{11} \mathrm{~A}$. The rating of the symptoms expressed in the systemic leaves of Nicotiana benthamiana and green pepper are indicated on the right; + = mosaic symptoms appearance, $-=$ no symptoms, $+/-=$ faint or minute mottle. TPD3-6249 could not infect plants, shown as "not infected" (n.i.). 
To generate a green fluorescent protein (GFP)-expressing PMMoV, the cDNA for an ER-localized GFP $(14,33)$ was inserted into the genome of three PMMoV species with essentially the previously described strategy (29), in the middle of the movement protein gene just below the CP subgenomic promoter, concatenating to the $3^{\prime}$ terminus of the genome of Tobacco mild green mottle virus Japanese strain (22) instead of $3^{\prime}$ UTR in itself. The first of the PMMoV-based infectious cDNA clones expressing the GFP gene was constructed using pTPC4350 as the base and designated pTPBG. The second and third were prepared using pTPJ and pTPA with the same strategy, to yield pTPJBG and pTPABG, respectively

The viruses derived from the in-vitro-transcribed RNAs were named by removing the prefix " $p$ " from the corresponding plasmids. Accordingly, TPC4350, equivalent to the wild type, was derived from pTPC4350.

Inoculation of plants with infectious transcripts. Infectious PMMoV transcripts were made with T7 RNA polymerase (Promega Corp., Madison, WI) from the pTP-series plasmids linearized with BamHI (17). In vitro transcripts were suspended in $10 \mathrm{mM}$ sodium phosphate buffer $(\mathrm{pH} \mathrm{7.0)}$ ) and inoculated onto the lower four true leaves of young green pepper seedlings ( Capsicum annuun) at the eight-true-leaf stage with carborundum as an abrasive. Inoculated plants were grown at $25^{\circ} \mathrm{C}(16 \mathrm{~h}$ of light $)$ in an artificially controlled growth chamber (Koito-Kogyo, Yokohama, Japan) to ensure systemic infection. For Nicotiana tabacum plants, transcripts were inoculated onto the single fifth true leaf when its length reached between 7.5 and $9 \mathrm{~cm}$, as previously described by Atkinson and Matthews (2).

Protoplast experiments. RNA transcripts were inoculated into protoplasts prepared from suspension-cultured tobacco cells (BY2) by electroporation with a Gene Pulser (Bio-Rad Laboratories, Hercules, CA). They were cultured exactly according to the protocol described previously (32). Protoplasts were harvested at 8 and $20 \mathrm{~h}$ postinoculation (hpi) for RNA and protein analyses, respectively.

Double-antibody sandwich enzyme-linked immunosorbent assay. Virus accumulation in pepper plants was assayed by a double-antibody sandwich enzyme-linked immunosorbent assay (DAS-ELISA) system based on a published method (4) with some modifications, including a specific antibody to PMMoV (35). Three pepper plants were inoculated with each of the transcripts. Four upper uninoculated leaves, corresponding to the sixth to ninth true leaves, were harvested from each plant at 10 days postinoculation (dpi), which exhibited the severest mosaic symptoms in the case of TPC4350. Serial dilutions (10-fold) of the sap (wt/vol) with $50 \mathrm{mM}$ phosphate buffer ( $\mathrm{pH} 7.0)$ containing $0.1 \%$ (wt/vol) PVP-40, 0.5\% (wt/vol) bovine serum albumen, $1 \mathrm{mM}$ EDTA, and $0.1 \%$ (vol/vol) $\beta$-mercaptoethanol were prepared to measure quantities of virus accumulated in the leaves. Virus accumulation in plants was assessed by comparing the dilutions with a standardized calibration curve in which purified PMMoV was serially diluted in the same way, starting from $100 \mu \mathrm{g} / \mathrm{ml}$. Three replicates of each sap dilution were placed into three wells of the ELISA plates, to allow the mean value to be calculated. The absorbance at $405 \mathrm{~nm}$ was determined for each well using an ELISA plate reader (Model 550; Bio-Rad Laboratories). The reader was auto-zeroed using sample-free buffer as a base value control. The final level of virus accumulation from each transcript was scored according to the mean absorbance at $405 \mathrm{~nm}$ from three plants.

RNA analysis. RNAs from protoplasts were extracted with TRIzol LS (Invitrogen, Carlsbad, CA) according to the manufacturer's instructions. For Northern blot analysis, total RNAs were glyoxylated, separated on a $1 \%$ agarose gel, then transferred to Hybond-N+ membrane (GE Healthcare Bio-sciences, Piscataway, NJ). After being cross-linked with UV illumination at 1,200 $\times$ $100 \mu \mathrm{J} / \mathrm{cm}^{2}$ using a Funa-UV-Crosslinker (Funakoshi, Tokyo), the membranes were prehybridized for at least $1 \mathrm{~h}$ in UltraHyb (Ambion, Austin, TX) at $68^{\circ} \mathrm{C}$. Hybridization was performed with digoxigenin-labeled riboprobes (Roche Diagnostics, Penzberg, Germany) corresponding to the 3' terminal $200 \mathrm{nt}$ of the PMMoV $3^{\prime}$ UTR derived from the cDNA plasmid clone, in which cDNA corresponding to above nucleotides were inserted between $\mathrm{T} 7$ for positive-sense RNA promoter and T3 for negative-sense RNA promoter, designated as pT73P3. Digoxigenin-labeled riboprobes were put onto the membrane at $68^{\circ} \mathrm{C}$ according to the manufacturer's instructions. Chemifluorescence was detected with using a digital imaging analyzer (Vasa-Doc; Bio-Rad Laboratories).

Specific amplification of transgene-derived GFP mRNA and GFP-tagged viral genomes by reverse-transcriptase (RT)-PCR was performed as follows. Central portions ( $2 \mathrm{~mm}$ in diameter) of GFP spots induced by TPBG, TPJBG, and TPABG were punched out under an epifluorescence stereomicroscope and subjected to total RNA extraction as described above. Disks of the same diameter were punched from outside the GFP spots on the same inoculated leaves or from mock-inoculated leaves. cDNAs were synthesized with Ready-to-Go RT-PCR Beads (GE Healthcare Bio-Sciences) with both oligo(dT) $)_{12-16}$ and a 20-nt oligonucleotide complementary to nucleotides 6159 to 6178 of PMMoV genome as primers. PCR primers used to amplify the GFP mRNA sequence were 5'-ATGAAGACTAATCTTTTTCTCTTTCTCATC-3', corresponding to the $\mathrm{N}$ terminus of GFP (part of the signal peptide), and 5'-TTAAAGCTCATGTTTGTATAGTTCATC-3', corresponding to the $\mathrm{C}$ terminus (including the ER retention signal). Primers used to amplify actin mRNA were 5'-ATGGCAGACGGTGAGGATATTCA-3' and 5'-GCCTTTGCAATCCACATCTGTTG-3'. Amplification was carried out with ExTaq DNA polymerase (Takara Bio, Tokyo) for 29 cycles (for GFP mRNA) or 40 cycles (for actin mRNA) of $94^{\circ} \mathrm{C}$ for $30 \mathrm{~s}, 55^{\circ} \mathrm{C}$ for $30 \mathrm{~s}$, and $72^{\circ} \mathrm{C}$ for $1 \mathrm{~min}$. Each PCR reaction $(50 \mu \mathrm{l})$ contained cDNAs derived from the total RNA equivalent to 0.1 GFP spot. Each PCR band was detected using a digital imaging analyzer (Vasa-Doc; Bio-Rad Laboratories).

Protein analysis. Total proteins were extracted with the protocol as previously described by Kubota et al. (18). After debris were removed, the supernatants were boiled and mixed with an equal volume of $20 \%$ glycerol containing $0.02 \%$ bromophenol blue. To detect CP or GFP, total protein was separated by 15 or $12.5 \%$ sodium dodecyl sulfate polyacrylamide gel electrophoresis, and transferred onto a polyvinylidene diflouride membrane (Millipore, Billerica, MA). The membrane was treated with primary antibodies against PMMoV CP (35) or GFP (Clontech, Mountain View, CA) and horseradish peroxidase-conjugated goat anti-rabbit immunoglobulin G secondary antibody (GE Healthcare Bio-Sciences). Immunoreactive bands were visualized by enhanced chemiluminescence (ECL) (GE Healthcare Bio-Sciences). The signals on the membrane were detected using a digital imaging analyzer (Vasa-Doc; Bio-Rad Laboratories).

Transgenic plants. To analyze PTGS suppressor activity in which PMMoV mutants possess the GFP-silenced $N$. tabacum cv. Samsun line (G3Sm2), the GFP-expressing tobacco line (G3Sm1), and the transgenic $N$. benthamiana plant constitutively expressing GFP (G3Nb3) were used as described previously (18). Inoculated tobacco plants (N. tabacum) were kept at $30^{\circ} \mathrm{C}$ for $16 \mathrm{~h}$ in the light and $25^{\circ} \mathrm{C}$ for $8 \mathrm{~h}$ in the dark, to ensure the systemic infection of PMMoV because tobacco plants are not natural hosts for the virus and not fully infected throughout a plant under $25^{\circ} \mathrm{C}$ conditions.

Observation of GFP fluorescence. GFP signals were observed under an epifluorescence stereomicroscope (MZ-FLIII; Leica Microsystems, Wetzlar, Germany) equipped with a filter set (GFP Plant; Leica Microsystems) and a CCD camera (DC500; Leica Microsystems). Plants were irradiated with the mercury lamp of the epifluorescence stereomicroscope through the GFP filter (450 to $490 \mathrm{~nm}$ ) and photographed with a CCD camera (DC500; Leica 
Microsystems) equipped with a lens (Zoom-Micro Nikkor 70 to $180 \mathrm{~mm}$; Nikon, Tokyo) and a band-path filter (500 to $550 \mathrm{~nm}$, Omega Optical, Brattleboro, VT) to reduce red autofluorescence.

\section{RESULTS}

PMMoV mutants. The attenuated phenotype of ToMV $\mathrm{L}_{11} \mathrm{~A}$ has been ascribed to three amino acid changes in the $130 \mathrm{~K}$ replication protein, Cys to Tyr at 349, Asn to Asp at 760, and Gly to Arg at 895 . An alignment of the $130 \mathrm{~K}$ protein between ToMV and PMMoV indicated that the corresponding positions of the PMMoV 130K protein are Met, Gly, and Arg, respectively. Therefore, we introduced single-base substitutions into the wild-type PMMoV and prepared two kinds of mutant viruses, TPJ and TPA, whose $130 \mathrm{~K}$ protein had an amino acid change from Met to Tyr at position 348 and from Gly to Asp at 762, respectively (Fig. 1).

The 3' UTR of PMMoV contains three consecutive pseudoknots, each composed of two double-helical segments, like those of ToMV. ToMV mutants with a mutation in the central pseudoknot infected plants systemically without causing symptoms in tobacco (31). Therefore, we introduced deletions into the pseudoknot region of PMMoV (Fig. 1): TPD3-6197 lacking the 5' proximal pseudoknot, TPD3-6207 lacking one of the two doublehelical segment of the central pseudoknot in addition to the $5^{\prime}$ pseudoknot, TPD3-6219 lacking the 5' and central pseudoknot, and TPD3-6249 lacking all the three pseudoknots.

Symptoms caused by mutant viruses and their multiplication in plants. Infectivity of the PMMoV mutants constructed was examined by inoculating the corresponding in vitro transcripts on green pepper plants. When the homogenates of the inoculated and upper leaves of the pepper plants at 14 dpi were inoculated on a local lesion host ( $N$. tabacum cv. Xanthi nc), numerous necrotic lesions were formed, except for the case of TPD3-6249. Because TPD3-6249 did not replicate in tobacco BY2 protoplasts, we concluded that the deletion of the three pseudoknots resulted in replication defectiveness (data not shown). The other five mutants were replication competent and spread systemically on pepper plants.

The five replication-competent mutants caused different symptoms on green pepper. TPJ caused no systemic symptoms (Fig. 2) even 4 weeks after inoculation (data not shown). When inoculated with TPA, green pepper plants displayed no or extremely weak symptoms, very faint or minute mottle, occasionally accompanied with very mild waves near the main and peripheral veins at 7 to 10 dpi. Similarly, N. benthamiana plants exhibited very mild mottle symptoms upon TPA infection but no symptoms upon TPJ infection (data not shown). The pepper plants infected with TPD3-6197 displayed severe mosaic symptoms on the top leaves which were indistinguishable from symptoms caused by TPC4350. In the case of TPD3-6207 or TPD3-6219, a mild mosaic or a mottle-like symptom was observed. This is in contrast to the observation that the ToMV mutants, in which the central pseudoknot in the 3' UTR was disrupted, infected asymptomatically on tobacco and tomato plants, as previously described (31).

To know whether there was a correlation between the severity of the mosaic symptoms and the amount of virus accumulated in the leaves, we measured accumulation levels of the mutants by DAS-ELISA (Fig. 2). The mosaic leaves of the TPC4350-infected pepper plants contained the virus at a level of $\approx 9.3 \mathrm{mg} / \mathrm{g}$ of leaves on average. The corresponding leaves of the TPJ- or TPA-infected plants contained a low level of virus at $\approx 3.5 \mathrm{mg} / \mathrm{g}$. In the case of the 3' UTR deletion mutants, accumulation levels of TPD3-6207 and TPD3-6219 were as low as 0.4 and $1.3 \mathrm{mg} / \mathrm{g}$, respectively, despite the obvious mosaic symptoms. TPD3-6197 caused typical mosaic symptoms, but its accumulation was $\approx 5.5 \mathrm{mg} / \mathrm{g}$, approximately half of the TPC4350 level. These data showed that the severity of the mosaic symptoms did not necessarily reflect the accumulation level of the virus. It appears that the virus encodes a factor responsible for the development of the mosaic symptoms, and that this factor was located on the $130 \mathrm{~K}$ replication protein itself.

Virus multiplication in tobacco protoplasts. To know whether the different levels of virus accumulation in the mosaic leaves is related to the ability of the viruses to replicate at the single-cell level, we examined multiplication of the PMMoV mutant at 8 and 20 hpi in tobacco protoplasts. When the PMMoV genomic RNA was analyzed by Northern blot analysis, $1 \mu \mathrm{g}$ of total RNAs from protoplasts was applied on the gel. For detection of a minus-strand RNA from each mutant, $3 \mu \mathrm{g}$ of total RNA was used. In Western blot analysis, $3 \mu \mathrm{g}$ of crude proteins extracted were added on the gel. Intensities of detected bands under a digital imaging analyzer were normalized with that of rRNAs in each, and then relative values of accumulated RNAs and CP were calculated based on a corresponding area of mock inoculation as background, which were exhibited as decimals, with the value for TPC4350 as 1.00 of its intensity. Accumulation levels of the genomic and the minus-strand RNAs of TPJ and TPA were similar to those of TPC4350 (Fig. 3). Accumulation of the $\mathrm{CP}$ for
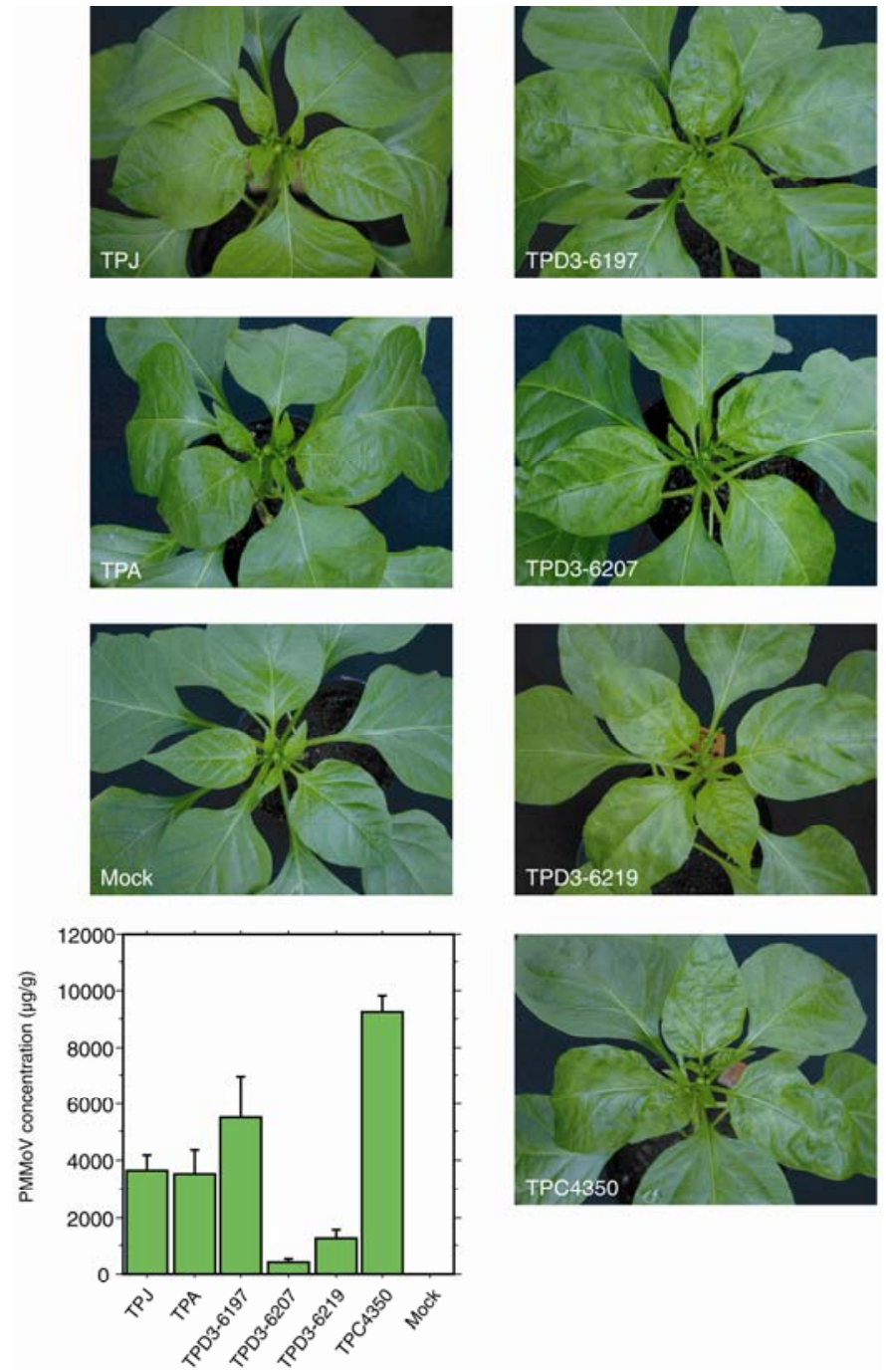

Fig. 2. Mosaic symptoms on the upper leaves of the green pepper plants infected with each mutant virus. Green pepper plants were inoculated with a virus at the eight-leaf stage and photographed at 10 days postinoculation (dpi). The graph indicates the amounts of the viruses accumulated at $10 \mathrm{dpi}$ in the four upper leaves of three green pepper plants inoculated with each virus, respectively. The value in each plant was determined by calculation with three replicates of each sap dilution into three wells of the enzyme-linked immunosorbent assay plates, and then statistically analyzed the mean value from three plants in each virus for preparation of the graph. 
the respective viruses was almost in parallel to that of the genomic and minus-strand RNAs. Therefore, TPJ and TPA multiplied similarly to the wild-type at the single-cell level. In contrast, RNA multiplication of TPD3 series mutants with a deletion in the pseudoknot region was limited at an approximate range from 10 to $70 \%$ levels against those of TPJ, TPA, and TPC4350. The CP accumulation of TPD3-series mutants appeared to reflect the RNA accumulation of the corresponding mutants, although the difference of the $\mathrm{CP}$ accumulation between the mutants was relatively low. Therefore, the relationship between mosaic symptoms and the replicability of the causative viruses were not correlated.

Relationship between the asymptomatic phenotype and defective PTGS suppressor activity. The above results indicate that the $130 \mathrm{~K}$ replication protein gene of PMMoV controls the expression of mosaic symptoms on the upper leaves. Because the Cysto-Tyr mutation in the $130 \mathrm{~K}$ protein of ToMV $\mathrm{L}_{11} \mathrm{~A}$ lowers the suppressor activity (18), we examined whether TPJ with the corresponding mutation is a suppressor-defective mutant. For this purpose, we first tried a reversal-of-silencing assay with $N$. benthamiana (3); however, when wild-type PMMoV was infected, the top leaves did not develop, so that the suppression of the silencing could not be evaluated. Then, we used a GFP-silenced tobacco line, G3Sm2 (18); we reported that, when ToMV was inoculated onto G3Sm2, GFP fluorescence was restored in the virus-rich yellow tissues of the mosaic leaves by the action of the suppressor, whereas $\mathrm{L}_{11} \mathrm{~A}$ did not have such activity.

When TPC4350 was inoculated onto the single fifth true leaf of G3Sm2, the GFP signal was detected at 4 or 5 dpi along with class I to III veins of the third to fifth upper leaves counted from the inoculated leaf (Fig. 4A), in which GFP expression had been silenced at the time of inoculation. Thereafter, the region in which GFP fluorescence was restored gradually broadened from the veins to the mesophyll tissues for 2 to 3 days. Further increase was not observed, due to the fact that $N$. tabacum is not a natural host for PMMoV; therefore, the virus could not fully infect throughout the plant, although the virus initially can invade systemic leaves and establish an asymptomatic infection $(8,17$, 23). Once GFP silencing was prevented by the virus, GFP fluorescence remained until the leaves became senescent.

When TPJ was inoculated on G3Sm2, GFP fluorescence was not restored in the upper leaves (Fig. 4A), although extremely faint GFP fluorescence occasionally was detected in the major vein of some upper leaves. This occurred only at an early stage of infection and GFP expression was again silenced within a few days. In the upper leaves of TPA-inoculated G3Sm2, in contrast, GFP fluorescence was restored, although its intensity was weaker than that seen in the TPC4350-infected leaves showing vein clearing (Fig. 4A).

To confirm that TPJ spread systemically in G3Sm2 without restoring GFP expression, extracts of the upper leaves were subjected to protein analysis and compared with those of TPAand TPC4350-infected plants (Fig. 4B). High levels of CP were detected mainly in the veins of leaf tissues in TPC4350-infected tobacco, where GFP protein also was detected at high levels. TPA-infected leaf tissues also contained both CP and GFP, but at lower levels than TPC4350-infected leaves. In the TPJ-infected G3Sm2 leaves, CP accumulated at a level similar to that in the TPA-infected leaves, whereas GFP did not increase upon infection. Therefore, TPJ was defective in the function to suppress PTGS in tobacco plants.

Next, we examined whether the mutation in the $130 \mathrm{~K}$ protein gene of TPJ also was associated with the suppressor-defective phenotype in green pepper and tobacco. For this purpose, we adopted an assay that we previously used for the analysis of the
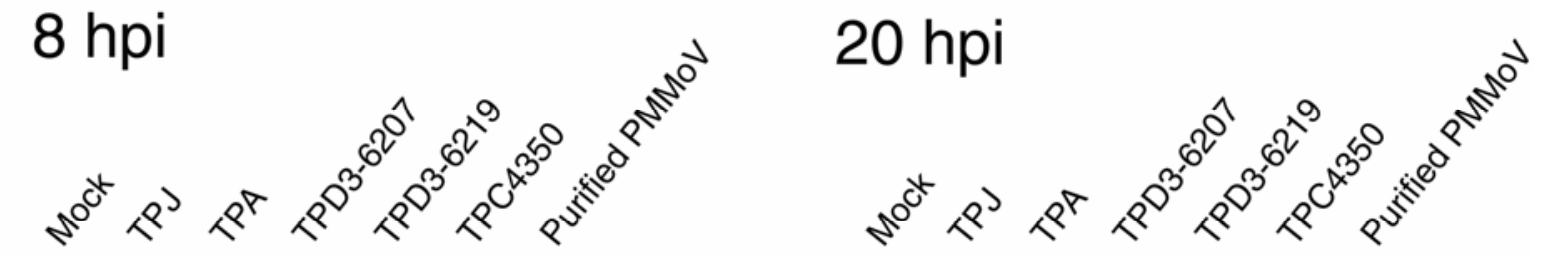

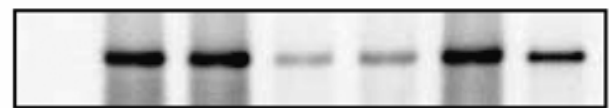

$\begin{array}{lllll}1.03 & 1.10 & 0.14 & 0.26 & 1.00\end{array}$

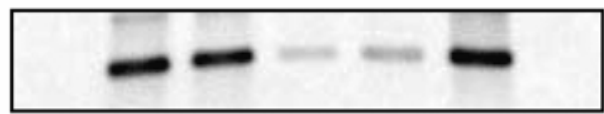

$\begin{array}{lllll}1.00 & 0.90 & 0.10 & 0.20 & 1.00\end{array}$

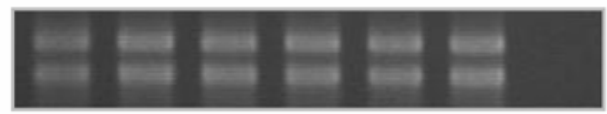

rRNA

(-) vg RNA

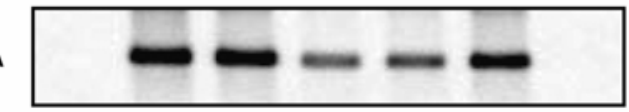

$\begin{array}{lllll}1.21 & 1.11 & 0.53 & 0.59 & 1.00\end{array}$
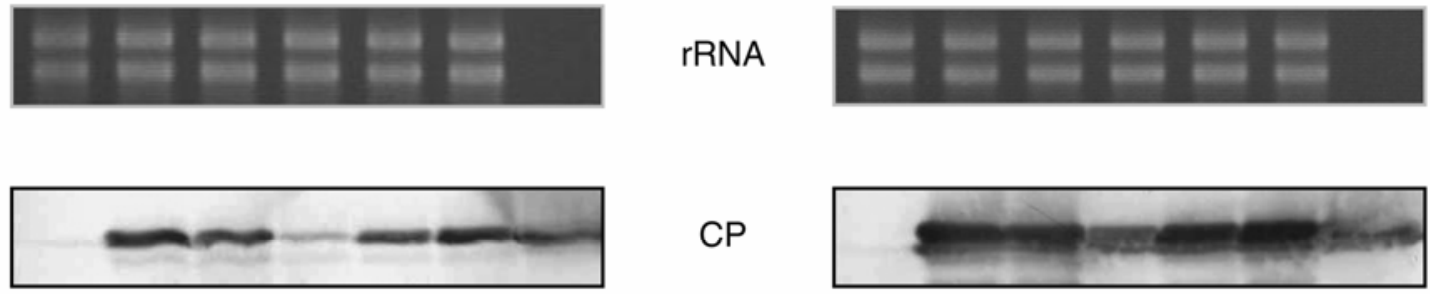

$\begin{array}{lllll}0.86 & 0.76 & 0.20 & 0.56 & 1.00\end{array}$
$\mathrm{CP}$

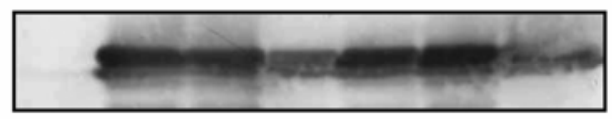

$\begin{array}{lllll}0.95 & 0.88 & 0.73 & 0.91 & 1.00\end{array}$

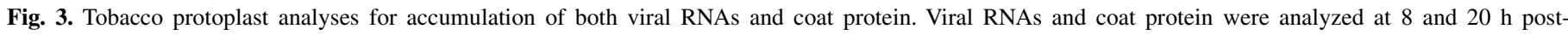

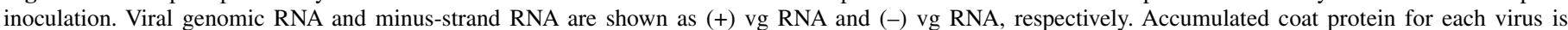

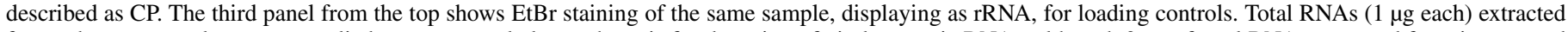

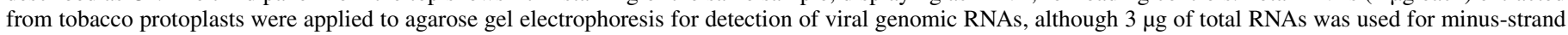

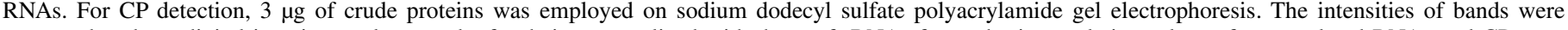

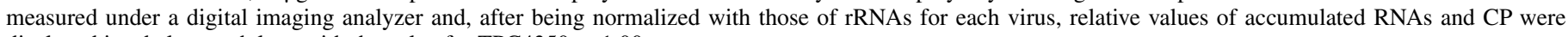
displayed just below each lane with the value for TPC4350 as 1.00. 
suppressor activity of ToMV; namely, GFP-tagged, suppressoractive ToMV formed round-shaped GFP spots on the inoculated leaves of tobacco, whereas GFP-tagged, suppressor-defective ToMV formed ring-shaped GFP spots (18). In the central portion of the ring-shaped GFP spots, PTGS occurred against the GFP sequences derived from both virus and the transgene. We prepared three GFP-tagged constructs, TPBG, TPJBG, and TPABG (Fig. $5 \mathrm{~A})$. TPBG carried the wild-type replication protein gene, whereas TPJBG and TPABG had the mutation as in TPJ and TPA, respectively. When inoculated on G3Sm1, a GFP-expressing tobacco, TPJBG formed ring-shaped GFP spots (Fig. 5B, arrowheads), whereas TPBG and TPABG exhibited round-shaped GFP spots, as expected. A decrease in GFP fluorescence in the center of the TPJBG-induced spots was observed from 4 or 5 dpi, after which the central regions enlarged. The GFP fluorescence from the center of the ring-shaped spots was less intense compared with fluorescence outside the spots, suggesting that transgenederived GFP expression was simultaneously silenced, although the centers ("in") of both TPBG and TPABG have not been reduced but, instead, enlarged, comparing the value for each "out" as 1.00 of its intensity as being understandable from numbers shown in Figure 5C. RT-PCR analysis showed that the decline of GFP fluorescence in the center of the ring-shaped spots, which

\section{A}
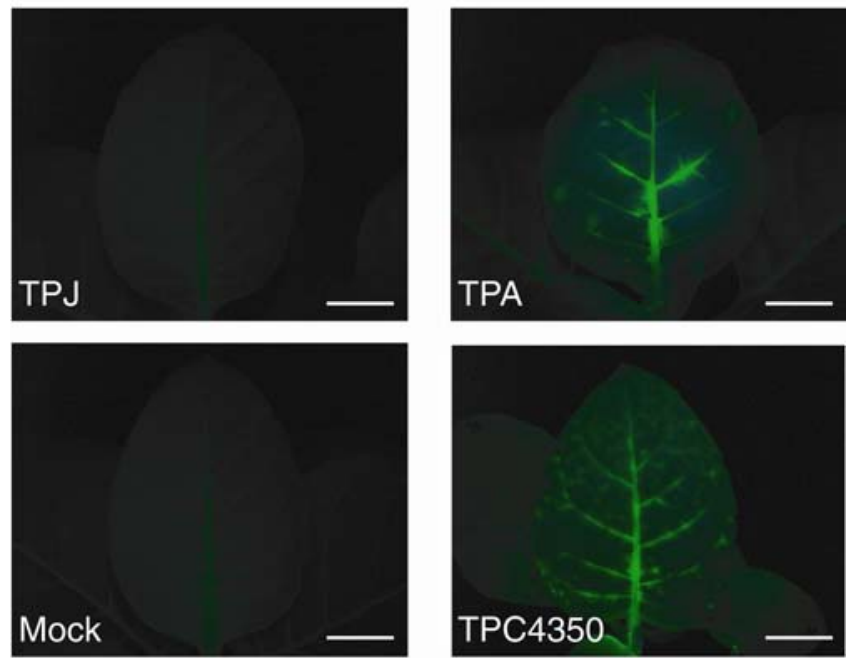

B

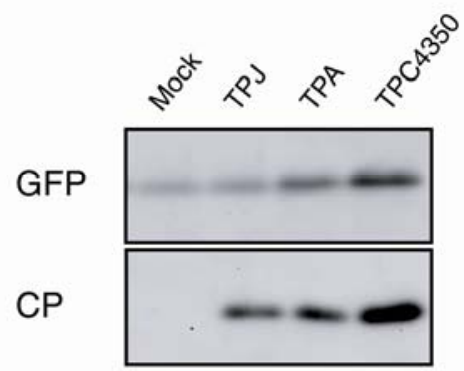

Fig. 4. Suppression of green fluorescent protein (GFP) silencing in G3Sm2 infected with Pepper mild mottle virus and its derivatives. A, GFP fluorescence patterns on the fifth upper leaf from the inoculated leaf. Plants were grown at $30^{\circ} \mathrm{C}$ and photographed at 14 days postinoculation. Bars represent $5 \mathrm{~cm}$. B, Western blot analyses for GFP and coat protein (CP) in the leaf veins. Proteins extracted from $1 \mathrm{mg}$ of leaf tissues were separated by sodium dodecyl sulfate polyacrylamide gel electrophoresis for the GFP analysis, whereas crude proteins from $0.25 \mathrm{mg}$ of leaf tissues were used for $\mathrm{CP}$ analysis. No increase in GFP protein was detected beyond leaky signals as a basal level in mock- or TPJ-infected leaves, whereas GFP protein was abundantly detected in TPA- and TPC4350-infected leaves. appeared only upon TPJBG infection, occurred together with a corresponding decrease in GFP mRNA.

This indicates that the decrease in GFP fluorescence in the center of the TPJBG-induced spots was associated with a decrease in transgene-derived GFP mRNA. These observations indicate that virus-targeting PTGS initiated but virus-encoded

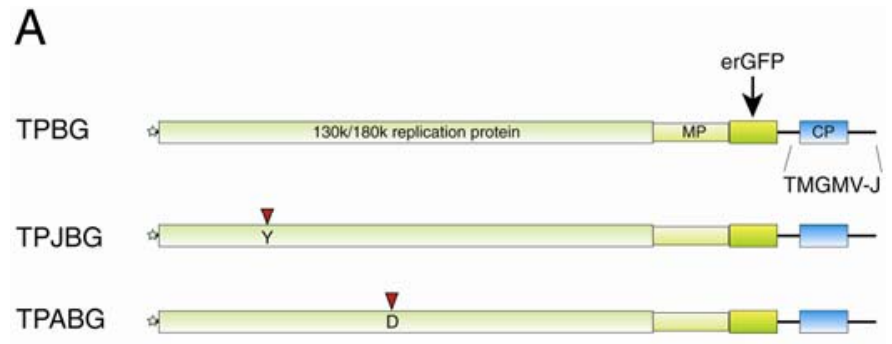

B
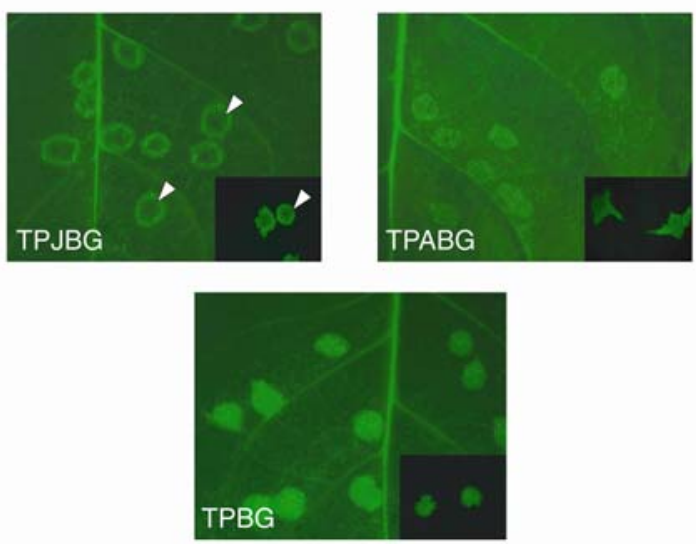

C

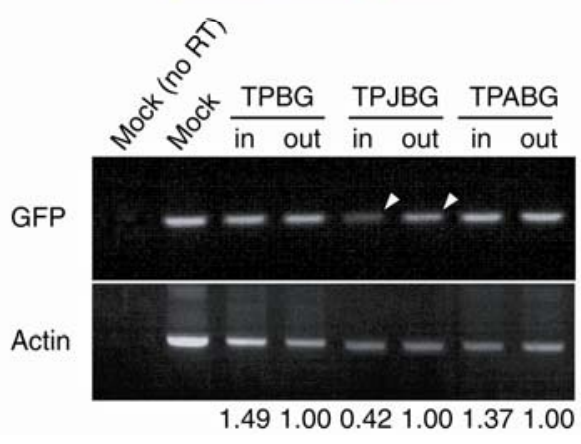

Fig. 5. Observation of posttranscriptional gene silencing suppression in the inoculated leaf of G3Sm1, a transgenic tobacco constitutively expressing green fluorescent protein (GFP). A, Schematic representation of ER-localized GFP (erGFP)-tagged Pepper mild mottle virus and derivatives. Substitutions in TPJBG and TPABG are in the position identical to TPJ and TPA, respectively (Fig. 1). B, GFP spots formed on an inoculated leaf of both G3Sm1 and green pepper (a small black square at the lower right in each picture). Arrowheads indicate the central regions of the GFP spots formed by TPJBG infection, where the expression of the resident GFP gene was silenced. Inoculated plants were kept at $30^{\circ} \mathrm{C}$ and pictures were taken at 5 days postinoculation. $\mathbf{C}$, Decrease in GFP mRNA in the central regions of TPJBG-induced GFP spots (compare the bands shown by arrowheads). RNAs were extracted to use as templates for reverse-transcription polymerase chain reaction (RT-PCR) from the center of GFP spots ("in") on leaves inoculated with TPBG, TPJBG, and TPABG. In lanes indicated as "out" in each, RNAs extracted from out of GFP spots were employed, in the area constitutionally expressing GFP and not invaded by viruses carrying a GFP gene. Paired lanes indicated at the top were derived from the same leaves. In the control "Mock (no RT)," PCR was performed with "Mock" sample without reverse transcription. Intensities of detected bands under a digital imaging analyzer were normalized with that of actin in each, which were calculated based on a corresponding area of mock inoculation as background; then, relative values for "in" of GFP mRNA, displayed just below each lane, were exhibited as decimals, with the value for "out" of each virus as 1.00 of its intensity. 
suppressor blocked the completion of the PTGS. However, the mutation in TPJBG could not block it; therefore, PTGS was established against the virus genome and the homologous GFP mRNA sequence.

Finally, these three constructs were inoculated on pepper plants. TPJBG formed ring-shaped GFP spots, whereas TPBG and TPABG formed round spots, as in tobacco, indicating that the mutation in the $130 \mathrm{~K}$ protein gene causing the Met-to-Tyr change also resulted in a suppressor-defective phenotype in pepper (Fig. $5 \mathrm{~B}$, small black square at the lower right in each picture). Our results indicate that the loss of systemic mosaic symptoms on green pepper plants by TPJ would be related to the lack of PTGS suppressor activity in itself. It follows that the development of mosaic symptoms during PMMoV infection of green pepper could be controlled by the PTGS suppressor activity encoded in the virus, but viral accumulation is not involved in PTGS suppressor activity.

\section{DISCUSSION}

Reversed genetic analyses of ToMV have shown that there are at least two kinds of mutations which result in asymptomatic infection in tobacco; one causes an amino acid change in the replication protein, originally found in attenuated strains $\mathrm{L}_{11}$ and $\mathrm{L}_{11} \mathrm{~A}$ and associating with a defective suppressor activity $(18,24)$, and the other is disruption of the central one of the three consecutive pseudoknots in the $3^{\prime}$ UTR (31). The replication protein mutants $\mathrm{L}_{11}$ and $\mathrm{L}_{11} \mathrm{~A}$ similarly multiply to the wild-type $\mathrm{L}$ at earlier stages of infection, but their final accumulation level is one-fifth to one-sixth of the wild-type level (25). In the case of $3^{\prime}$ UTR mutants, both replication and accumulation levels also were reduced, resulting in asymptomatic infection on tobacco and tomato. Therefore, there was an idea that severity of mosaic symptoms caused by ToMV might be related to the level of virus accumulation and there might be a threshold level for the development of mosaic symptoms.

In this study, we examined whether the findings with ToMV mutants in tobacco and tomato were true for PMMoV in pepper. From the analyses of the TPD3 series mutants with a mutation in the pseudoknot region, we found that the $3^{\prime}$ UTR mutations caused reduced levels of multiplication but did not establish asymptomatic infection. Further, the comparisons of the accumulation levels between the wild-type and mutant viruses showed that the accumulation levels were not correlated with the severity of the mosaic symptoms, as exemplified by the fact that TPJ infected without symptoms, accumulating much higher than TPD36207 and TPD3-6219 (Fig. 2). This suggests that mosaic symptom development is not simply a result of virus accumulation over a threshold level but that there is another mechanism controlling the development of the systemic symptoms, in which the $130 \mathrm{~K}$ protein is involved.

Several viruses cause mosaic symptoms on the systemic leaves of infected plants, resulting in patterns of light and dark green areas (15) which are specific to particular combinations of virus and host plant species. The development and structure of mosaics caused by TMV in infected tobacco leaf tissue have been observed and described in detail (2). For instance, mosaics first appear in the developing, sink leaves less than $1.5 \mathrm{~cm}$ in length at the time of systemic invasion, so that the leaf becomes dark greenish in one half and yellowish in the other. Yellow-green tissues allow significant virus replication and accumulation (8). Microscopical analyses showed that the chloroplasts in cells invaded by the virus were fatally damaged, causing leaf tissues to appear yellow $(15,20)$. Although these data represent histological and pathological observations, the hypothesis recently has been proposed that the underlying mechanism involves PTGS, as a host defense system to viral infections. PTGS may play an important role in the formation of mosaic patterns on the leaf, which results from the activity of the host PTGS combating the suppressor activity encoded by the virus genome $(27,37,38)$, including the development of mosaic symptoms (21). Therefore, it would be assumed that the intensity of the PTGS suppressor activity encoded by the virus is important for the production of mosaics on the leaf. We already indicated in a previous article that wildtype ToMV possesses a PTGS suppressor activity on the $130 \mathrm{~K}$ replication protein (18). Mutant viruses with a single amino acid substitution at the end of the methyltransferase domain toward the helicase domain either lost or decreased the suppressor activity, resulting in an asymptomatic phenotype (18). In the present study, it was demonstrated that PMMoV also possesses the PTGS suppressor activity on its $130 \mathrm{~K}$ replication protein, like ToMV (Figs. 4 and 5).

Apparently, the replication protein of tobamoviruses, including PMMoV, has dual function: one is viral replication and the other is RNA silencing suppression. Thus, it is likely that the mutations in TPJ and TPA may affect both viral replication and PTGS suppression. Hence, the experiment done with protoplast in this study was important to assay only virus replication in a PTGSfree environment. In a previous report described by Silhavy et al. (30), the mutant Tomato bushy stunt virus belonging to the genus Tombusvirus that lacks a functional PTGS suppressor accumulates to a similar level as that of wild-type virus, suggesting that PTGS may not be a limiting factor for virus replication in protoplasts. Similarly, it is in the protoplast experiment that we have demonstrated that the mutations in TPJ and TPA, attenuated phenotype, only affected the PTGS suppression activity of replication protein, whereas viral accumulation leves were relatively parallel between them including that of wild-type virus. Consequently, the suggested conclusion is that viral pathogenicity is controlled by the PTGS suppression activity of the PMMoV replication protein, but not the viral accumulation. However, TPA, whose suppressor activity apparently was higher than that of TPJ, infected pepper plants with no or extremely mild symptoms and accumulated at a level similar to TPJ. Therefore, the factor participating in symptom development may not be solely the PTGS suppressor.

To examine the PTGS suppressor activity of PMMoV, we first carried out a reversal-of-silencing assay by using transgenic $N$. benthamiana harboring a GFP gene $(\mathrm{G} 3 \mathrm{Nb} 3)$ (18), in which the expression was systemically silenced by agroinfiltration of an inverted-repeat construct of the GFP gene, according to a previously described method (3). However, when PMMoV was inoculated onto a systemically GFP-silenced plant at the five- to sixtrue-leaf stage, the plant showed severe yellowing and curling symptoms at 7 to 10 dpi before the restoration of GFP expression, which ordinarily were seen on $N$. benthamiana (data not shown). As a second trial, agro-infiltration of the same plant type was tested using Agrobacterium carrying the $130 \mathrm{~K}$ replication protein gene of PMMoV, according to the protocol established by Erickson et al. (6). However, the $130 \mathrm{~K}$ replication protein of PMMoV failed by this method, whereas both the $130 \mathrm{~K}$ replication protein of ToMV and HC-Pro of Potato virus Y (PVY) successfully suppressed PTGS against GFP (data not shown). Finally, recombinant viruses expressing GFP (TPBG and its derivatives) provided a piece of evidence, showing PTGS suppressor activity on an inoculated leaf of G3Sm1 tobacco plant (Fig. 5). Throughout these experiments, we became aware that the intensity of the PTGS suppressor activity encoded by a virus genome would differ according to virus species. This might be tightly associated with the severity of mosaic symptoms on a host plant because, in nature, mosaic symptoms are characteristic of each combination of virus and host (15). Taken together, it is likely that the suppressor activity of PMMoV is weaker on Nicotiana plants than those of ToMV, PVY, and similar viruses.

A cross-protection strategy based on an attenuated virus originally was used to protect crops from infection with severe 
virus strains in the field. Although cultivars carrying a gene conferring resistance to virus infection have largely replaced this approach, the appearance of resistant viruses over time means that the original attenuated virus strategy still may be useful $(10,35)$. ToMV- $\mathrm{L}_{11} \mathrm{~A}$ has been used successfully as an attenuated strain to protect tomato plants in Japan (26). However, selection of appropriate mild or attenuated strains requires a laborious screening of strains with trained eyes; therefore, a logical strategy for the creation of attenuated strains is desirable. The mutation in the replication protein of $\mathrm{L}_{11} \mathrm{~A}$ results in a suppressor-defective phenotype, as we showed previously (18). When this mutation was introduced to the PMMoV replication gene, the mutant virus TPJ acquired the suppressor-defective phenotype, showing the loss of the activity for mosaic symptom expression (Figs. 2 and 4). This amino acid substitution, Met-to-Tyr at residue 349 of the $130 \mathrm{~K}$ replication protein of $\mathrm{PMMoV}$, plays a crucial role in both the PTGS suppressor activity and mosaic symptom expression. When the substitution was changed to cysteine, the original amino acid in the ToMV $130 \mathrm{~K}$ replication protein, severe systemic mosaic symptoms appeared, showing that PTGS suppressor activity actually was maintained on the G3Sm2 tobacco plant (data not shown). Our finding that the mutation in the replication protein of $\mathrm{L}_{11} \mathrm{~A}$ can be applied to PMMoV indicates that, in tobamoviruses, the suppressor gene is the target to create an attenuated strain.

\section{NOTE}

This article includes corrections for faults described in the previous paper published by Yoon et al. (42).

\section{ACKNOWLEDGMENTS}

Part of this study was supported by a grant-in-aid of "the research project for utilizing advanced technologies in agriculture, forestry and fisheries" administered by the Ministry of Agriculture, Forestry and Fisheries in Japan.

\section{LITERATURE CITED}

1. Agrios, G. N. 2005. Plant Pathology. Fifth Edition. Elsevier Academic Press, London.

2. Atkinson, P. H., and Matthews, R. E. F. 1970. On the origin of dark green tissue in tobacco leaves infected with tobacco mosaic virus. Virology 40:344-356.

3. Brigneti, G., Voinnet, O., Li, A.-X., Ji, A.-H., Ding, A.-W., and Baulcombe, D. C. 1998. Viral pathogenicity determinants are suppressors of transgene silencing in Nicotiana benthamiana. EMBO J. 17:6739-6746.

4. Clark, M. F., and Adams, A. N. 1977. Characteristics of the microplate method of enzyme-linked immunosorbent assay for the detection of plant viruses. J. Gen. Virol. 34:475-483.

5. Ding, X. S., Liu, J., Cheng, N.-H., Folimonov, A., Hou, Y.-M., Bao, Y., Katagi, C., Carter, S. A., and Nelson, R. S. 2004. The tobacco mosaic virus $126-\mathrm{kDa}$ protein associated with virus replication and movement suppresses RNA silencing. Mol. Plant-Microbe Interact. 17:583-592.

6. Erickson, F. L., Holzberg, S., Calderon-Urrea, A., Handley, V., Axtell, M., Corr, C., and Baker, B. 1999. The helicase domain of the TMV replicase proteins induces the $\mathrm{N}$-mediated defense response in tobacco. Plant $\mathrm{J}$. 18:67-75.

7. Fauquet, C. M., Mayo, M. A., Maniloff, J., Desselberger, U., and Ball, L. A. 2005. Virus Taxonomy. Eighth Report of the International Committee on Taxonomy of Viruses. Elsevier Academic Press, London.

8. Fulton, R. W. 1951. Superinfection by strains of tobacco mosaic virus. Phytopathology 41:579-592.

9. Garcia-Arenal, F. 1988. Sequence and structure at the $3^{\prime}$ end of the $\mathrm{U}_{2^{-}}$ strain of Tobacco mosaic virus, a histidine accepting tobamovirus. Virology 167:201-206.

10. Garcia-Luque, I., Ferrero, M. L., Rodriguez, J. M., Alonso, E., de la Cruz, A., Sanz, A. I., Vequero, C., Serra, M. T., and Diaz-Ruiz, J. R. 1993. The nucleotide sequence of the coat protein genes and $3^{\prime}$ non-coding regions of two resistance-breaking tobamoviruses in pepper shows that they are different viruses. Arch. Virol. 131:75-88.

11. Goto, H., Itai, T., and Sato, S. 1997. Selection of attenuated viruses for control of sweet pepper mosaic disease caused by Tobacco mosaic virus pepper strain and Cucumber mosaic virus, and their effects. Bull. Oita Prefect. Agric. Res. Cent. 27:79-122.

12. Goto, T. 1984. Utilization of attenuated strain for control of pepper mosaic disease caused by tobacco mosaic virus. Crop Prot. 38:349352 .

13. Hamilton, A. J., and Baulcombe, D. C. 1999. A species of small antisense RNA in posttranscriptional gene silencing in plants. Science 286:950952.

14. Haseloff, J., Siemering, K. R., Prasher, D. C., and Hodge, S. 1997. Removal of a cryptic intron and subcellular localization of green fluorescent protein are required to mark transgenic Arabidopsis plants brightly. Proc. Natl. Acad. Sci. USA 94:2122-2127.

15. Hull, R. 2002. Matthews' Plant Virology, 4th ed. Academic Press, London.

16. Kiho, Y., and Nishiguchi, M. 1984. Unique nature of an attenuated strain of tobacco mosaic virus: Autoregulation. Microbiol. Immunol. 28:589599.

17. Kirita, M., Akutsu, K. Watanabe, Y., and Tsuda, S. 1997. Nucleotide sequence of the Japanese isolate of pepper mild mottle tobamovirus (TMV-P) RNA. Ann. Phytopathol. Soc. Jpn. 63:373-376.

18. Kubota, K., Tsuda, S., Tamai, A., and Meshi, T. 2003. Tomato mosaic virus replication protein suppresses virus-targeted posttranscriptional gene silencing. J. Virol. 77:11016-11026.

19. Leathers, V., Tanguay, R., Kobayashi, M., and Gallie, D. R. 1993. A phylogenetically conserved sequence within the viral $3^{\prime}$ untranslated RNA pseudoknots regulates translation. Mol. Cell Biol. 13:53315347.

20. Matthews, R. E. F. 1991. Plant Virology, 3rd ed. Academic Press, London.

21. Moor, C. J., Sutherland, P. W., Forster, R. L. S., Gardner, R. C., and MacDiarmid, R. M. 2001. Dark green islands in plant virus infection are the result of posttranscriptional gene silencing. Mol. Plant-Microbe Interact. 14:939-946.

22. Morishima, N., Ido, T., Hamada, H., Yoshimoto, E., Mizumoto, H., Takeuchi, S., Kiba, A., Hikichi, Y., and Okuno, T. 2003. Infectious in vitro transcripts from a cDNA clone of tobacco mild green mosaic tobamovirus and its biological activity in host and nonhost plants and in their protoplasts. J. Gen. Plant Pathol. 69:335-338.

23. Nagai, Y. 1987. Production of C-1421, an attenuated mutant of pepper strain of tobacco mosaic virus. Ann. Phytopathol. Soc. Jpn. 53:168174.

24. Nishiguchi, M., Kikuchi, S., Kiho, Y., Ohno, T., Meshi, T., and Okada. Y. 1985. Molecular basis of plant viral virulence; the complete nucleotide sequence of an attenuated strain of tobacco mosaic virus. Nucleic Acids Res. 13:5585-5590.

25. Nishiguchi, M., Motoyoshi, F., and Oshima, N. 1990. Comparison of virus production in infected plants between an attenuated tomato strain $\left(\mathrm{L}_{11} \mathrm{~A}\right)$ and its wild strain (L) of tobacco mosaic virus. Ann. Phytopathol. Soc. Jpn. 56:691-694.

26. Oshima, N. 1981. Control of tomato mosaic disease by attenuated virus. Jpn. Agric. Res. Qt. 14:222-228.

27. Pruss, G., Ge, X., Shi, X. M., Carrington, J. C., and Vance. V. B. 1997. Plant viral synergism: The potyviral genome encodes a broad-range pathogenicity enhancer that transactivates replication of heterologous viruses. Plant Cell 9:859-868.

28. Rietveld, K., Linschooten, K., Pleij, C. W. A., and Bosch, L. 1984. The three-dimensional folding of the tRNA-like structure of tobacco mosaic virus RNA. A new building principle applied twice. EMBO J. 3:26132619.

29. Shivprasad, S., Pogue, G. P., Lewandowski, D. J., Hidalgo, J., Donson, J., Grill, L. K., and Dawson, W. O. 1999. Heterologous sequences greatly affect foreign gene expression in Tobacco mosaic virus-based vectors. Virology 255:312-323.

30. Silhavy, D., Molnár, A., Lucioli, A., Szittya, G., Hornyik, C., Tavazza, M., and Burgyán, J. 2002. A viral protein suppresses RNA silencing and binds silencing-generated, 21- to 25-nucleotide double-stranded RNAs. EMBO J. 21:3070-3080.

31. Takamatsu, N., Watanabe, Y., Meshi, T., and Okada, Y. 1990. Mutational analysis of the pseudoknot region in the $3^{\prime}$ noncoding region of tobacco mosaic virus RNA. J. Virol. 64:3686-3693.

32. Tamai, A., Kubota, K., Nagano, H., Yoshii, M., Ishikawa, M., Mise, K., and Meshi, T. 2003. Cucumovirus- and bromovirus-encoded movement functions potentiate cell-to-cell movement of tobamo- and potexviruses. Virology 315:56-67.

33. Tamai, A., and Meshi, T. 2001. Tobamoviral movement protein transiently expressed in a single epidermal cell functions beyond multiple plasmodesmata and spreads multicellularly in an infection-coupled manner. Mol. Plant-Microbe Interact. 14:126-134.

34. Tanguay, R. L., and Gallie, D. R. 1996. Isolation and characterization of the 102-kilodalton RNA-binding protein that binds to the $5^{\prime}$ and $3^{\prime}$ translational enhancers of tobacco mosaic virus RNA. J. Biol. Chem. 
271:14316-14322.

35. Tsuda, S., Kirita, M., and Watanabe, Y. 1998. Characterization of a pepper mild mottle tobamovirus strain capable of overcoming the $L^{3}$ genemediated resistance, distinct from the resistance-breaking Italian isolate. Mol. Plant-Microbe Interact. 11:327-331.

36. Van Belkum, A., Abraham, J. P., Pleij, C. W. A., and Bosch, L. 1985. Five pseudoknots are present at the 204 nucleotides long $3^{\prime}$ non-coding region of tobacco mosaic virus RNA. Nucleic Acids Res. 13:7673-7686.

37. Voinnet, O. 2005. Induction and suppression of RNA silencing: Insights from viral infections. Nat. Rev. Genet. 6:206-220.

38. Voinnet, O., Pinto, Y. M., and Baulcombe, D. C. 1999. Suppression of gene silencing: A general strategy used by diverse DNA and RNA viruses of plants. Proc. Natl. Acad. Sci. USA 96:14147-14152.
39. Watanabe, Y., Morita, N., Nishiguchi, M., and Okada, Y. 1987. Attenuated strains of tobacco mosaic virus, reduced synthesis of a viral protein with a cell-to-cell movement function. J. Mol. Biol. 194:699704.

40. Yoneyama, S. 1988. Control of P strain of tobacco mosaic virus in sweet pepper. (2) Effects of soil fumigation with methyl bromide in spring season. Kanto-Tosan Plant Prot. Soc. 35:53-55. (In Japanese)

41. Yoneyama, S. 1988. Control of P strain of tobacco mosaic virus in sweet pepper. (3) Effects of soil fumigation with methyl bromide in summer season. Kanto-Tosan Plant Prot. Soc. 35:56-57. (In Japanese)

42. Yoon, J. Y., Ahn, H. I., Kim, M., Tsuda, S., and Ryu, K. H. 2006. Pepper mild mottle virus pathogenicity determinants and cross protection effect of attenuated mutants in pepper. Virus Res. 118:23-30. 